'improvement' was mentioned in 4 cases, while arthritis was improved in 6 patients, 4 of which had secondary SS.

In terms of extraglandular manifestations (e.g cryoglobulinemic vasculitis, interstitial nephritis) 18 were reported. 16 (88.8\%) patients received Rituximab (one of them in combination with Belimumab), while $2(11.1 \%)$ were treated with TNFinhibitors. $11.1 \%$ and $61.1 \%$ of them received concurrent treatment with csDMARDS and steroids, respectively. $55.5 \%$ and $11.1 \%$ of the patients were csDMARDs- and biologics- experienced, respectively. Extraglandular manifestations responded well in the majority $(83.3 \%)$ of the patients, with the remaining having partial or late response. SS and arthritis 'improvement' was mentioned in 5 and 1 patients, respectively.

Table 1 Biologic treatments used for overlapping autoimmune conditions in SS patients. csDMARDs: conventional synthetic Disease Modifying Antirheumatic Drugs, NA: Not Applicable, Joints: improvement of arthritis. ^SS response was defined as clinical improvement of sicca symptomatology or improvement in ESSDAI. Improvement of arthritis was reported separately. $\uparrow$ cases of secondary Sjogren's syndrome ( 3 cases co-existing with RA and one case with SLE)

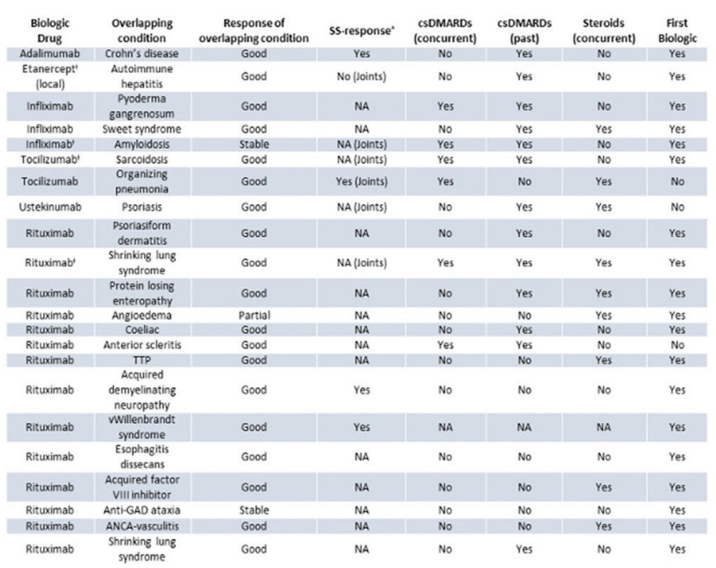

Conclusions: Treatment with biologic DMARDs, sometimes accompanied by steroids, appears to be beneficial also in treating overlapping autoimmune diseases as well as some extraglandular manifestations in SS patients.

Disclosure of Interest: None declared

DOI: 10.1136/annrheumdis-2018-eular.7200

\section{THU0355 DAMAGE ACCRUAL AND MORTALITY RATES AMONG DIFFERENT AGE GROUPS IN A COHORT OF PATIENTS WITH LUPUS}

G.A. Ramirez, B. Tejera-Segura, R. Lopez, A. Rahman, D.A. Isenberg. Centre for Rheumatology, University College London (UCL) Hospital NHS Foundation Trust, London, UK, London, UK

Background: Systemic lupus erythematosus (SLE) is a chronic, multi-system autoimmune disease, characterised by relapses and remissions, which eventually can lead to progressive, irreversible organ damage accrual. SLE-related damage implies increased morbidity and impaired quality of life. In addition, it constitutes itself a risk factor for the development of further damage and associates with early mortality. Age at disease onset has been postulated to affect the presenting clinical phenotype, the rate and likelihood of accruing damage and eventually mortality risk. Early-onset SLE patients have been reported to present with a more aggressive disease, especially in the first years after diagnosis. However, longterm data from large cohorts are scanty.

Objectives: To assess retrospectively the mortality and damage accrual rates in a large cohort of patients with SLE.

Methods: Demographic, clinical and laboratory data from 692 consecutive patients with SLE (631 females, 61 males), diagnosed according to the revised American College of Rheumatology (ACR) criteria in a single tertiary referral centre, were retrospectively analysed focusing on age of diagnosis. Damage accrual was assessed according to the Systemic Lupus Erythematosus
International Collaborating Clinics (SLICC)/ACR damage index (SDI). Cox's regression analysis, chi-square test, Kruskal-Wallis test and ANOVA were employed as appropriate.

Results: Thirty-three out of 692 patients were diagnosed with SLE before the age of 12,172 between 12 and 20, 443 between 21 and 50 and 44 after 50 years of age. As previously reported, a female preponderance was more evident in the central part of the age spectrum $(p=0.015)$. Nephritis and decreased complement were more frequent in patients with early-onset SLE $(p=0.001$ and $p=0.033$ respectively), serositis in the central age groups ( $p=0.025)$, and arthritis in lateonset patients $(p=0.002)$. Neuropsychiatric manifestations were less frequent in patients aged $>50$ years $(p=0.013$; table 1$)$. The global incidence rate for any damage was 48.36 per 1000 persons-years, whereas the death incidence rate was 8.54 per 1000 persons-years. Late-onset SLE associated with a higher risk of damage accrual $(H R=1.63, p=0.024)$ and of death $(H R=6.22, p<0.001)$. However there were no significant differences in the time to first damage, to death afte diagnosis and to death after the development of the first damage item, according to the age of diagnosis.

Conclusions: Younger patients with SLE show a distinct clinical phenotype, but share an accelerated accrual of morbidity and a higher risk of early mortality with patients of older age. Identifying age-specific predictors of disease severity will be of outstanding importance to improve long-term survival rates and patients' quality of life.

\section{REFERENCES}

[1] Lopez R, et al. Rheumatology 2012.

[2] Ambrose N, et al. Lupus 2016.

[3] Lim LSH, et al. Arthritis Care Res, 2017.

Disclosure of Interest: None declared

DOI: 10.1136/annrheumdis-2018-eular.3896

\section{THU0356 SYNERGISTIC EFFECT OF CUMULATIVE CORTICOSTEROID DOSE AND IMMUNOSUPPRESSANTS ON AVASCULAR NECROSIS IN PATIENTS WITH SYSTEMIC LUPUS ERYTHEMATOSUS}

${ }^{1}$ H.-H. Kwon, S.-Y. Bang ${ }^{1}$, S. Won ${ }^{2}$, Y. Park ${ }^{2}$, J.-H. Yi ${ }^{3}$, Y.B. Joo ${ }^{1}$, H.-S. Lee ${ }^{1}$, S. C. Bae ${ }^{1} .{ }^{1}$ Department of Rheumatology, Hanyang University Hospital For Rheumatic Diseases; ${ }^{2}$ Clinical Research Center for Rheumatoid Arthritis (CRCRA), Seoul; ${ }^{3}$ Department of Internal medicine, Hanyang University Guri Hospital, Guri, Korea, Republic of Ireland

Background: Avascular necrosis (AVN) is one of the most common organ damage in patients with systemic lupus erythematosus (SLE) and often causes serious physical disability.

Objectives: The aims of this study were to investigate clinical risk factors associated with symptomatic AVN and to analyse their synergistic effects in a large SLE cohort in Korea.

Methods: Patients with SLE were enrolled and followed from 1998 to 2014 in the Hanyang BAE Lupus cohort, in whom damage was measured annually according to the Systemic Lupus International Collaborating Clinics/American College of Rheumatology Damage Index. AVN was confirmed by imaging study if patients had symptoms. To determine risk factors for AVN, clinical, laboratory, and therapeutic variables were analysed by logistic regression. Relative excess risk due to interaction (RERI), attributable proportion (AP), and synergy index (S) were calculated to measure interactions between significant variables.

Results: Among 1,219 SLE patients, symptomatic AVN was the most common type of musculoskeletal damage $(10.8 \%, n=132)$. SLE patients with AVN showed an earlier onset age, demonstrated AVN more commonly in conjunction with certain other clinical manifestations such as renal and neuropsychiatric disorders, and received significantly higher total cumulative corticosteroid dose and immunosuppressive agents than did patients without AVN. However in multivariable analysis, only two variables including use of a cumulative corticosteroid dose greater than $20 \mathrm{~g}$ (odds ratio $(\mathrm{OR}) 3.08, \mathrm{p}=0.005$ ) and use of immunosuppressants including cyclophosphamide or mycophenolate mofetil (OR 4.34, $\mathrm{p}=0.002$ ) remained as significant risk factors for AVN. Patients with cumulative corticosteroid dose $>20 \mathrm{~g}$ and immunosuppressants use had

\begin{tabular}{|c|c|c|c|c|c|c|c|c|c|c|c|}
\hline $\begin{array}{l}\text { Age group } \\
\text { (years) }\end{array}$ & $\mathrm{N}$ & $\begin{array}{c}\text { Females } \\
(\%)\end{array}$ & $\begin{array}{l}\text { Nephritis } \\
(\%)\end{array}$ & $\begin{array}{c}\text { Neurological inv. } \\
(\%)\end{array}$ & $\begin{array}{c}\text { Arthritis } \\
(\%)\end{array}$ & $\begin{array}{l}\text { Rash } \\
(\%)\end{array}$ & $\begin{array}{c}\text { Photosensitivity } \\
(\%)\end{array}$ & $\begin{array}{l}\text { Oral ulcers } \\
\text { (\%) }\end{array}$ & $\begin{array}{c}\text { Serositis } \\
(\%)\end{array}$ & $\begin{array}{c}\text { antiDNA } \\
(\%)\end{array}$ & $\begin{array}{c}\text { Reduced complement } \\
(\%)\end{array}$ \\
\hline$<12$ & 33 & 78.8 & 48.5 & 27.3 & 72.7 & 81.8 & 42.4 & 27.3 & 18.2 & 72.7 & 51.5 \\
\hline $12-20$ & 172 & 89.5 & 41.9 & 22.1 & 87.2 & 65.7 & 39.0 & 30.2 & 37.8 & 66.9 & 55.0 \\
\hline $21-50$ & 443 & 93.2 & 33.4 & 22.6 & 91.9 & 66.8 & 41.8 & 26.0 & 41.1 & 61.2 & 46.0 \\
\hline$>50$ & 44 & 86.4 & 11.4 & 6.8 & 93.2 & 54.5 & 27.3 & 22.7 & 27.3 & 63.6 & 31.8 \\
\hline
\end{tabular}


15.44-fold increased risk for AVN, compared with patients without these risk factors $(p<0.001)$. RERI, AP, and $S$, which define the strength of interactions between two risk factors, were 9.01 (95\% confidence interval (CI) 1.30-16.73), 0.58 (95\% Cl $0.36-0.81)$, and 2.66 (95\% Cl 1.42-4.99), respectively, supporting the presence of synergistic interactions in the development of symptomatic AVN in our Korean lupus cohort.

Abstract THU0356 - Table 1. Synergistic effect of total cumulative steroid dose and use of immunosuppressants in development of AVN in SLE patients

\begin{tabular}{|c|c|c|c|c|}
\hline & $\begin{array}{l}\text { No. patients } \\
\text { with AVN }\end{array}$ & $\begin{array}{l}\text { No. patients } \\
\text { without AVN }\end{array}$ & $\begin{array}{c}\mathrm{OR}^{\mathrm{a}} \\
(95 \% \mathrm{Cl})\end{array}$ & $\mathrm{p}$-value \\
\hline $\begin{array}{l}\text { Total cumulative steroid dose } \leq 20 \mathrm{~g} \\
\text { and immunosuppressants (-) }\end{array}$ & 11 & 314 & 1.00 & \\
\hline $\begin{array}{l}\text { Total cumulative steroid dose }>20 \mathrm{~g} \\
\text { and immunosuppressants (-) }\end{array}$ & 17 & 168 & $\begin{array}{c}3.08 \\
(1.40- \\
6.81)\end{array}$ & 0.005 \\
\hline $\begin{array}{l}\text { Total cumulative steroid dose } \leq 20 \mathrm{~g} \\
\text { and immunosuppressants }(+)\end{array}$ & 18 & 121 & $\begin{array}{c}4.34 \\
(1.99- \\
9.49)\end{array}$ & $<0.001$ \\
\hline $\begin{array}{l}\text { Total cumulative steroid dose }>20 \mathrm{~g} \\
\text { and immunosuppressants }(+)\end{array}$ & 64 & 134 & $\begin{array}{l}15.44 \\
(7.64- \\
31.19)\end{array}$ & $<0.001$ \\
\hline RERI & & & $\begin{array}{c}9.01 \\
(1.30- \\
16.73)\end{array}$ & \\
\hline AP & & & $\begin{array}{c}0.58 \\
(0.36- \\
0.81)\end{array}$ & \\
\hline$S$ & & & $\begin{array}{c}2.66 \\
(1.42- \\
4.99)\end{array}$ & \\
\hline
\end{tabular}

AVN: avascular necrosis; SLE: systemic lupus erythematosus; OR: odds ratio; $\mathrm{Cl}$ : confidence interval; RERI: relative excess risk due to interaction; AP: attributable proportion; S: synergy index

a. Odds ratios were adjusted for sex, age and disease duration.

Conclusions: An individual risk assessment for AVN development should be made prior to and during treatment for SLE, especially in patients with high-dose corticosteroid and immunosuppressant use regardless of clinical manifestations and disease activity.

Disclosure of Interest: None declared

DOI: 10.1136/annrheumdis-2018-eular.3438

\section{THU0357 FEATURES ASSOCIATED WITH LOSS TO FOLLOW-UP IN THE YEAR PRIOR TO DEATH IN PATIENTS WITH SYSTEMIC LUPUS ERYTHEMATOSUS: A RETROSPECTIVE ANALYSIS FROM A NATIONAL REFERRAL CENTRE}

${ }^{1}$ I. Padjen, M. Erceg ${ }^{2}$, M. Cerovec ${ }^{1}$, M. Bakula ${ }^{1}$, M. Mayer ${ }^{1}$, R. Stevanovic ${ }^{2}$, B. Anic ${ }^{1}$. 1 Department of Internal Medicine, Division of Clinical Immunology and Rheumatology, University Hospital Centre Zagreb and University of Zagreb, School of Medicine; ${ }^{2}$ Croatian Institute of Public Health, Zagreb, Croatia

Background: Loss to follow-up in the year prior to death may lead to underrecog nition and underreporting of systemic lupus erythematosus (SLE) as a cause of death of lupus patients.

Objectives: We aimed to assess the extent and features associated with loss to follow-up in the year prior to death in a group of 90 deceased SLE patients from our tertiary centre.

Methods: We retrospectively analysed 90 SLE patients (68 females) followed-up at our centre, deceased from 2002 to 2011 . Patients were $\geq 18$ years of age at death and fulfilled $\geq 4$ classification criteria of the American College of Rheumatology (ACR). The cause and place of death were identified by matching patient data from our department's SLE registry with data from the National Death Database. Patients were considered lost to follow-up in the year prior to death (LTF) if the time span between the last visit to our centre and death exceeded 1 year. Other patients were considered to be under regular follow-up (RGF).

An extensive set of parameters was compared between the LTF and RGF groups: demographics, ACR classification criteria, cumulative damage according to the Systemic Lupus International Collaborative Clinics (SLICC)/ACR index, as well as causes of death. Frequencies were compared using the chi-square and Fisher's exact test, and continuous variables using the t-test and Mann-Whitney U-test.

Results: We identified 35/90 patients in the LTF group (29 females). The time span between the last visit to our centre and death of LTF patients ranged from $>1$ to 3 years. Compared to the RGF group, LTF patients were diagnosed at a later age (mean $\pm S D$ : $54 \pm 15$ vs. $44 \pm 17$ years, $p=0.006$ ), while there was no difference in disease duration (median of 11 years, IQR of 5-15 years in the RGF group vs. median of 7 years, IQR of $5-15$ years in the LTF group, $p=0.285$ ). The
LTF and RGF groups did not differ in the count of ACR criteria (median of 5, IQR of 4-6 vs. median of 6, IQR of $5-7, \mathrm{p}=0.053$ ) and cumulative damage (median damage of 3 , IQR of $2-5$ vs. median of 5 , IQR of $3-8, p=0.068$ ).

Compared to the RGF group, LTF patients had a lower cumulative proportion of pericarditis (1/35 vs.16/55), proteinuria (10/35 vs. $30 / 55)$, hemolytic anaemia (1/ 35 vs. $10 / 55)$, thrombocytopenia (5/35 vs. $21 / 55)$ and Hughes syndrome (2/35 vs. $13 / 55)(p<0.05)$. Pulmonary damage and peripheral vascular damage were observed only in RGF patients (9/55 vs. $0 / 35, p=0.011$; and $8 / 55$ vs. $0 / 35$, $p=0.021$, respectively). LTF patients also had a lower proportion of cardiomyopathy $(7 / 35$ vs. $24 / 55, p=0.021)$

RGF patients died more frequently from active lupus compared to their LTF counterparts $(24 / 55$ vs. $2 / 35, p<0.001)$, while no difference was observed between the proportions of death from infections, cardiovascular diseases, malignancies and unknown causes (figure 1). SLE was reported in death certificates of 30/55 RGF patients compared to only $11 / 35$ LTF patients $(p=0.032)$. Compared to the RGF group, a lesser proportion of LTF patients died in the hospital (17/35 vs. $46 / 55$, $\mathrm{p}=0.004)$.

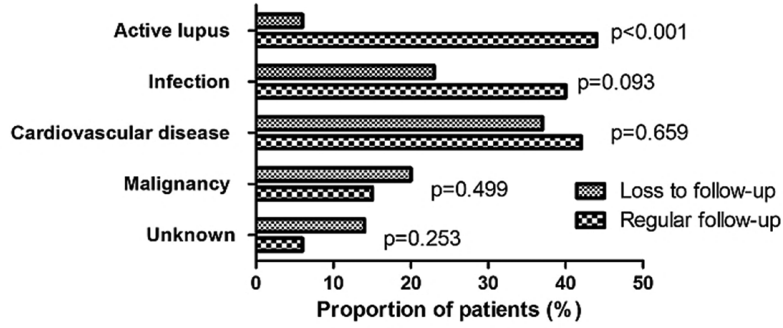

Abstract THU0357 - Figure 1. Causes of death according to follow-up status

Conclusions: A lower proportion of LTF patients exhibited features of active SLE over their disease course. This may have led to underrecognition of SLE as a contributor to death.

\section{REFERENCES}

[1] Calvo-Alen J, et al. Rheumatology 2005;44:1186-9.

[2] Abu-Shakra M, Novack V. J Rheumatol 2012;39:458-60.

Disclosure of Interest: None declared

DOI: 10.1136/annrheumdis-2018-eular.3567

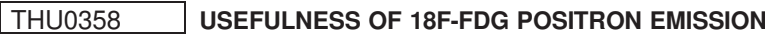 TOMOGRAPHY (PET) FOR LYMPHOMA DIAGNOSIS IN PATIENTS WITH PRIMARY SJÖGREN'S SYNDROME}

${ }^{1}$ J. Keraen, E. Blanc ${ }^{2}$, F. Besson ${ }^{3}$, M. Meyer ${ }^{3}$, V. Le Guern ${ }^{4}$, G. Nocturne', J. Henry ${ }^{1}$, R. Belkhir ${ }^{1}$, X. Mariette ${ }^{1}$, R. Seror ${ }^{1} .{ }^{1}$ Rheumatology, CHU du Kremlin Bicêtre, Kremlin Bicêtre; ${ }^{2}$ Nuclear imaging, Centre Chirugical Marie Lannelongue, Le Plessis Robinson; ${ }^{3}$ Nuclear imaging, CHU du Kremlin Bicêtre, Kremlin Bicêtre ${ }^{4}$ Internal Medicine, Cochin, Paris, France

Background: Primary Sjögren's syndrome (pSS) is the autoimmune disease having the highest risk of lymphoma. The differential diagnosis between benign and malignant lymphoproliferation is sometimes difficult. Among imaging procedures, 18F-FDG PET could be useful for that purpose.

Objectives: To compare 18F-FDG PET results between patients with and without lymphoma to identify PET pattern associated with lymphomas in pSS

Methods: Retrospective study conducted in 2 centres including pSS patients (according to ACR/EULAR 2016 criteria) who undergo 18F-FDG PET. We com pared PET abnormalities in patients with and without lymphoma, the PET having been done before any chemotherapy. Two independent readers analysed PET blind to lymphoma diagnosis. ESSDAI-PET score previously described by Cohen et al.[' was calculated.

Results: 45 patients were included; 15 had lymphoma: MALT ( $n=12)$, nodal marginal zone with plasmacytic differentiation $(n=2)$, diffuse large B-cell $(n=1)$. Patients with lymphoma had more frequently parotid gland swelling $(67 \%$ vs $20 \%$, $\mathrm{p}=0.003)$ and higher ESSDAI score (24 [13.5-29] vs $\left.9,^{5-20} \mathrm{p}=0.03\right)$, even after exclusion of lymphoma item $\left(19^{11-27}\right.$ VS $\left.9,{ }^{5-20} \mathrm{p}=0.03\right)$.

Compared to non-lymphoma patients, mean size $\left(45.5^{38-56} \mathrm{~mm}\right.$ vs. $40^{37-41} \mathrm{~mm}$ $\mathrm{p}=0.048$ ) and maximum standardised uptake value (SUVmax) of the parotid glands (5.6 [5-6.9] vs 3.8 [3.2-4.4]; $\mathrm{p}=0.001)$ were higher in lymphoma patients $53.3 \%$ of patients with lymphoma and $43.3 \%$ without lymphoma had lymph node FDG uptake, but neither their number nor their repartition or mean SUV differ between them. 